\title{
Alveolar rhabdomyosarcoma mimicking acute leukemia in an adolescent with deep venous thrombosis - case presentation
}

\author{
Daniela Voda ${ }^{1,2}$, Anca Corina Leonte ${ }^{1}$, Carmen Otelea ${ }^{1}$, Anca Maria Ilea ${ }^{1,2}$ \\ 1Emergency Children's Hospital, Brasov, Romania \\ ${ }^{2}$ Transilvania University, Brasov, Romania
}

\begin{abstract}
Rhabdomyosarcoma is the most common type of soft-tissue sarcoma, who invade and destroy nearby tissues. Rhabdomyosarcoma has defective myogenic differentiation and the alveolar subtype is the most aggressive, with hithgrade malignancy.

We present the case of a 13-years-old girl, with a history of pain, functional impairment, red skin colour and swollen of the left thigh. The investigations have shown thrombosis on the left femoral and iliac vein, thrombosis that resulted from genetic predisposition together with the mechanisme of paraneoplastic thrombosis. The imagistic investigation documented retroperitoneal adenopathic masses encompassing the large vesels, gluteal and perianal mases, solid mass in the pancreatic head and multiple bone nodular lesions. The nonhematopoietic malignancies has masquerading acute leukemia on the bone marrow aspirate with atypical blasts ( $80 \%$ from cells population). The histological examination of the biopsy sample following excision of the left inguinal lymphadenopaty have revealed the final diagnosis: Stage IV alveolar rhabdomyosarcoma, with bone marrow infiltration and multiple metastases (inguinal, pelvic, periaortic, perihepatic), pancreatic and orbital metastases.
\end{abstract}

Keywords: rhabdomyosarcoma, deep venous thrombosis, acute leukemia

\section{INTRODUCTION}

Rhabdomyosarcoma, malignant tumor with defective myogenic differentiation, has an aggressive behavior as it can invade and destroy nearby tissues resulting early dissemination. Is the most common type of soft-tissue sarcoma in pediatric age and is comprised of three subtypes: embryonal, alveolar and pleomorphic. Alveolar tumors is the second most common subtype and has a worse prognosis. Is usually found in adolescents and it used to occur in deep musculature of the extremities $(1,2,3)$. The symptomatology usually doesn't exist, unless a nerve is compressed. Precisely for this reason, the majority of the patients present from the beginning advanced disease with multiple metastases. The most frequent sites of metastases are: head, bones, lymph nodes and lung. Studies have shown that the metatases of alveolar subtype tends to involves the bones. On the other hand, metastases in the bone marrow, pancres or in the orbit are extremely rare and shows worse prognosis (3-6).

The histopatological examination confirm the diagnosis, and MRI can determinate the site of tumor origin, the extent of local invasion and the metastases. The prognosis is bad and the 5-year survival rate (after chemotherapy, radiotherapy and surgical therapy) is posible over the $80 \%$, but only if doesn't exist metastatic disease at the time of diagnosis $(1,7,8)$.

The association between malignancy and venous thrombotic disease is well known. Armand Trousseau was the first who proved the connection cancer thrombosis. Paraneoplastic thrombosis is based on procoagulant status result from the activity of tumoral tissue - like factors who are capable to activating the coagulation cascade and who releas the excess of thrombin. Also, in malignant tissues it was highlighted the procoagulant substances who activate the $\mathrm{X}$ factor $(9,10)$. 


\section{CASE REPORT}

We present the case of a 13-years-old girl, with a history of pain, functional impairment, red skin colour and swollen of the left thigh for 2 weeks ago. The family history and the pathological history were not relevant.

Clinical examination at the presentation revealed the following pathological changes: swollen of the left thigh (there was 6 centimeters between the circumference of the thights), local cyanosis and palpatory pain from the lef inguinal region to the left malleolar region; also was palpable one well-defined left inguinal lymphadenopathy (with approximate dimensions of $2 / 2 \mathrm{~cm}$ ). She was hospitalized under the suspicion of a deep venous thrombosis.

Laboratory investigations revealed: normocytic normochronic anemia (Hgb $9.8 \mathrm{~g} / \mathrm{dl}$, Hct 28.2\%), hyposideremie (serum iron $29 \mu \mathrm{g} / \mathrm{dl}$ ), hith level of lactate dehydrogenase (943 U/1) and increased level of inflammatory markers $(\mathrm{CRP}=1.3 \mathrm{mg} / \mathrm{dl}$, ERS $=85$ $\mathrm{mm} / 1 \mathrm{~h}, \mathrm{FIB}=623 \mathrm{mg} / \mathrm{dl})$.

We investigated the possible causes may be involved in the etiology of the thrombosis, like: hyperhomocysteinemia, vasculitis, antiphospholipid syndrom or neoplastic disease. So, paraclinical data for all these showed: normal homocystein levels, negative values for lupus aticoagulant, antiphospholipid antibodies, cardiolipin, antinuclear and antiDNA antibodis. The thrombophilia didn't fit with normal levels of protein $\mathrm{C}, \mathrm{S}$ and with genetic profile of thrombofilia that was associated with an increased risk of thrombosis. The genetic tests didn't show any mutation of the factor V Leiden, factor XIII V34L, factor II or MTHER C677T gene mutation). However, we found two homozygous mutation (MTHFR A 1298C and PAI-1 4G/4G) and an hyperactivity of factor VIII.

The abdominal ultrasound highlighted the thrombosis of the left femoral and iliac veins, abdominal and inguinal masses, multiples nodules with lymphadenopatic characteristics.

We performed a thoraco-abdomial CT scan with contrast who revealed, beside the thrombosis, the presence of extensive retroperitoneal adenopathic masses encompassing the large vessels (Fig. 1).

Abdominal and pelvic RMI examination with contrast documented new important changes: solid mass in the pancreatic head and also in the uncinate proces; multiple solid pancreatic lesions (Fig. 2); gluteal and perianal masses (Fig. 3); colecyst with inhomogeneous content and with biliary calculus; inhomogeneous bone structure with multiple confluent nodular lesions, the bones of the both femur and the pelvis being involved almost entirely.

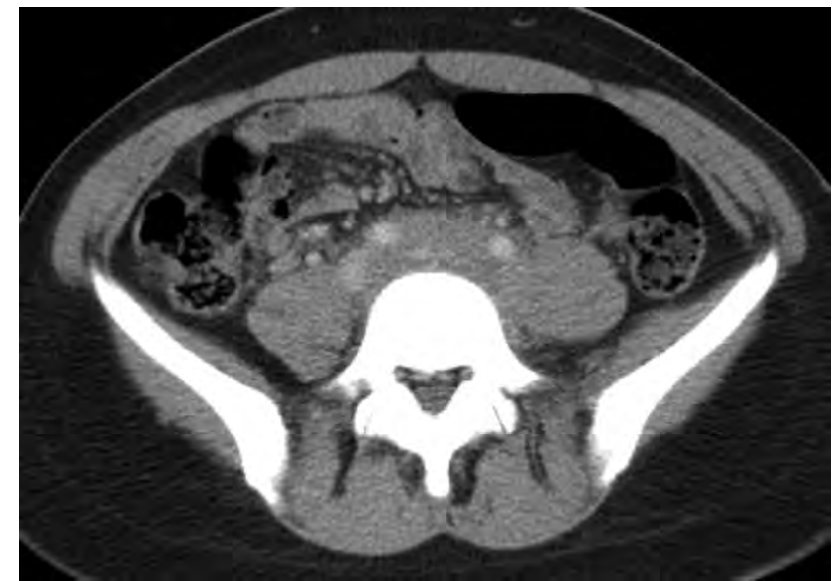

FIGURE 1. Retroperitoneal adenopathic masses with large vessel embedding; left femoral and iliac vein thrombosis, CT aspect

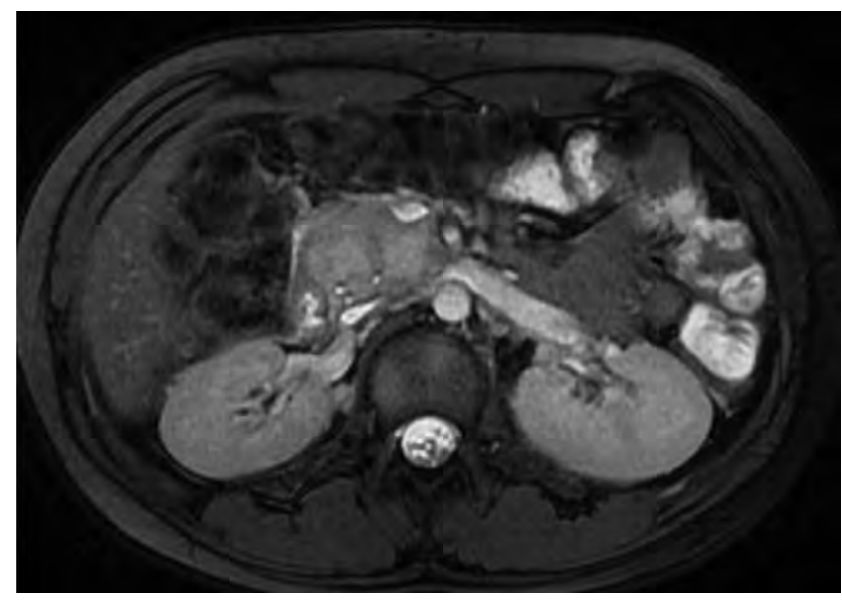

FIGURE 2. Pancreatic head mass, RMI aspect

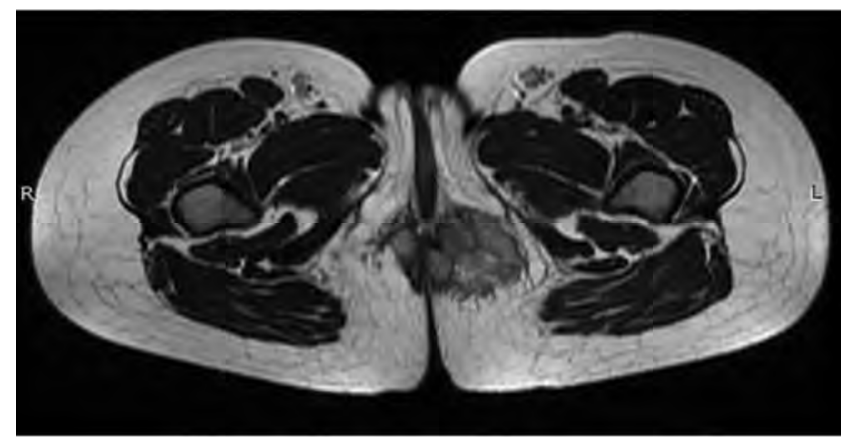

FIGURE 3. Gluteal and perianal masses, RMI aspect

Bone marrow aspirate revealed an important cellularity with atypical blasts cells population (approximately $80 \%$ ), a moderate amount of basophilic cytoplasm with vacuoles and 1-2 nucleolus. The distribution of blasts cells showed tendency to coalesce into medium and small groups or they were isolated (Fig. 4).

The histopatological examination of the biopsy sample (following excision of the left inguinal lymphadenopathy) and the immunohistochemistry highlighted an inguinal lymph node metastasis of an alveolar rhabdomyosarcoma. 


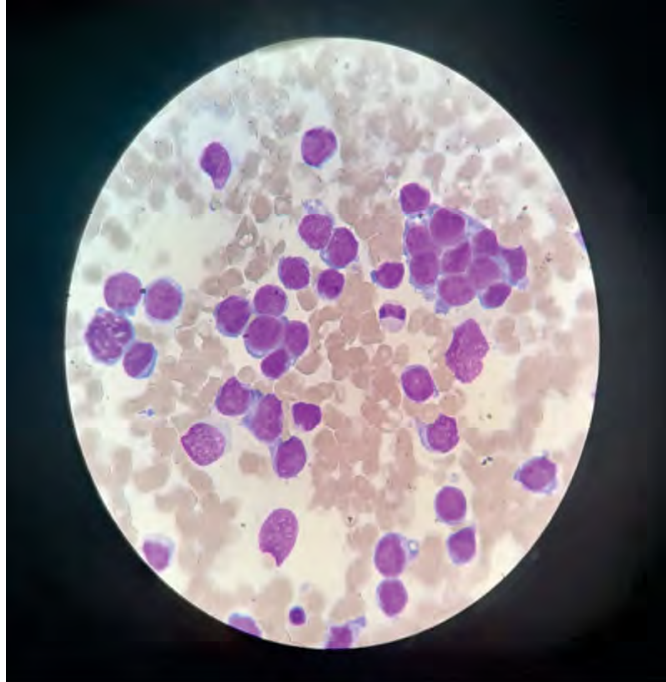

FIGURE 4. Bone marrow aspirate, May-Grünwald-Giemsa stain (MGG), x 100

Concerning all this pathological findings, the final diagnosis was made: stage IV alveolar rhabdomyosarcoma, with bone marrow infiltration and multiple metastases (inguinal, pelvic, periaortic, perihepatic) and pancreatic metastases.

Our pacient followed 4 cycles of chemotherapy according to the CWS 2009 protocol.

In evolution, the pacient associated elevated transaminase (ALT $460 \mathrm{U} / 1$, AST $86 \mathrm{U} / 1$ ) and amylase (622 U/l). It was performed an endoscopic retrograde colangiography who suggested an adenopathic obstruction of the common bile duct. It was necessary to placed an plastic biliary stent.

At the imagistic evaluation of the treatment response, a new metastasis was detected at the orbital region. The bone marrow aspirate didn't showed malignant cell. Due to the extent of tumor and to poor treatment response, the adjuvant radiotherapy was initiated for local control of the disease.

\section{DISCUSSIONS}

Alveolar rhabdomyosarcoal is a malignant tumor with myogenic differentiation and belongs to the small round cell tumors group. Is usually found in adolescents, is extremely aggressive and has a high rate of metastasis $(3,11)$.

In this case, due to an impressive deep vein thrombosis, il was nesessary to remove the other causes of thrombosis. The thrombophylia, protein $\mathrm{C}$ or S deficiency, antithrombin deficiency, vasculitis, antiphospholipid syndrome or hyperhomocysteinemia are some of the possible causes. All of this were excluded in our pacient.

The genetics of the thrombophilia plays an important role for estimating the risk. The gene mutations who were associated with an increased risk are: mutation of the factor V Leiden, factor II, factor XIII or MTHER C677T $(12,13)$. Our genetic tests didn't show any of this mutations, however, the patient had two homozygous mutations (MTHFR A1298 C and PAI-1 4G/4G). Reported data about the prevalence of this homozygous mutation are controversial. Based on several study, the PAI-1 4G/4G might be a risk factor, even more if is associated with other defects such as hyperhomocysteinemia, protein $\mathrm{C}$ or $\mathrm{S}$ deficiency or factor VIII hyperactivity - this also being present in our case (14). Regarding the MTHFR A1298C mutation, there was no study that could demonstrate the association with the thrombosis risk (13).

Returning to our case, we can conclude the fact that the patient has an genetic predisposition for thrombosis (due to the PAI-1 4G/4G mutation associated with factor VIII hyperactivity), over which overlapped the involved mechanisme for paraneoplastic thrombosis.

Another point of interest in this case is the bone marrow infiltration by the non-hematopoietic neoplasia. There are few case reports in the literature who showed that the rhabdomyosarcoma can mimic acute leukemia in childhood $(15,16)$. We highlight 4 other similar cases, beginning in adolescence, reported in the last 9 years. These cases showed a predisposition for male sex ( 3 boys and one girl), between 14 and 15 years old, and who completely mimic the acute leukemia from the beginning, both histologically and clinically. Bone marrow aspirate revealed, in all these cases, atypical blasts cells population and basophilic cytoplasm with vacuoles, cells that were also highlighted in our case. The differences in our report consist in following aspects: the pacient was female and the mimicking of acute leukemia was incomplete. There were no specific clinical criteria for acute leukemia, the bone marrow aspirate was the one who pleaded for acute leukemia. All these studies have shown that the bone marrow infiltration is uncommon, but when it appears can lead to misdiagnosis, delaying or even losing the diagnosis (17-20).

Studies have shown that the metastases in the bone marrow, pancreas or in the orbit are extremely rare and show worse prognosis (4-6).

\section{CONCLUSIONS}

The diagnosis for rhabdomyosarcoma is a challenge due to the insidious onset and the non-specific symptomatology. The tumor aggression and the early dissemination are reflected in the advanced disease that pacients present from the beginning, with multiple metastases. 
Due to this things, it becomes absolutely necessary that rhabdomyosarcoma be discussed each time when an intra-abdminal mass is highlighted to the investigations, in the case of atypical leukemia, or even when a neoplasm is suspected without being detected the primary site.

\section{REFERENCES}

1. Petra Balogh, Rita Bánusz, Monika Csóka et al. Primary alveolar rhabdomyosarcoma of the bone: Two cases and review oft he literature. Diagnostic Pathology. 2016; 11:99.

2. Ida Russo, Virginia Di Paolo, Carmelo Gurnari et al. Congenital rhabdomyosarcoma: A different clinical presentation in two cases. BMC Pediatrics. 2019; 18:166.

3. Debojyoti Sakar, Sayantal Ray, Manjary Saha et al. Alveolar rhabdomiosarcoma with multilple distal metastases. A case report and review of literature. BMJ Casse Reports. 2012; 2:10

4. Kim J, Ussher JG. Orbital rhabdomyosarcoma: A rare ophthalmic condition. Med. J. Aust. 2019 Nov; 211(9):398-399.

5. Ryan AL, Nagarajan L, Alessandri AJ et al. Rare pattern of relapse to the pancreas and bilateral extraocular muscles in paediatric alveolar rhabdomyosarcoma. J. Paediatr. Child Health. 2017 Apr; 53(4):419-421.

6. Córdoba Rovira, Inarejos Clemente. Childhood rhabdomyosarcoma. Radiologia. 2016 Nov - Dec; 58(6):481-490.

7. Yang QK, Li SL, Chen T et al. Clinicopathological characteristics and therapeutic effects of adult rhabdomyosarcoma patients. Zhonghua Zhong Liu Za Zhi. 2019 Nov 23; 41(11):873-877.

8. Koivusalo Al, Rintala RJ, Pakarinen MP. Combined management of perianal rhabdomyosarcoma with chemotherapy, radical surgery, and irradiation: A series of three consecutive children. J. Pediatr. Surg. 2019 Feb; 54(2):285-287.

9. Mizoguchi S, Sawai T, Hirota A, Yamamoto S et al. Trousseau's Syndrome Causing Refractory Deep Venous Thrombosis. Intern. Med. 2018 Feb 15; 57(4):623-626

10. Nowak-Göttl U, van Ommen H, Kenet G. Thrombophilia testing in children: What and when should be tested? Thromb. Res. 2018 Apr; 164:75-78

11. Jawad N, McHugh $\mathrm{K}$. The clinical and radiologic features of paediatric rhabdomyosarcoma. Pediatr. Radiol. 2019 Oct; 49(11):1516-1523.

12. Den Heijer M, Lewington S, Clarke R. Homocysteine, MTHFR and risk of venous thrombosis: A meta-analysis of published epidemiological studies. Journal of Thrombosis and Haemostasis. 2005; 3:292-299.
The particularity of the our case is given by mimicking the acute leukemia on the bone marrow aspirate, the thrombotic manifestation resulting from a paraneoplastic complication and, not least, by the sites of the metastases.

\section{Conflict of interest: none declared Financial support: none declared}

13. Mohammad Soleyman Soltanpour, Zahra Soheili, Ali Akbar Pourfathollah et al. The A1298C Mutation in Methylenetetrahydrofolate Reductase Gene and Its Association With Idiopathic Venous Thrombosis in an Iranian Population. LabMedicine. 2010 April; 42 (4).

14. Prabhudesai A, Shetty $S$, Ghosh $K$ et al. Investigation of Plasminogen Activator Inhibitor-1 (PAI-1) 4G/5G promoter polymorphism in Indian venous thrombosis patients: A case-control study. Eur. J. Haematol. 2017 Sep; 99(3):249-254.

15. Bernardo López-Andrade, Maria Antonia Duran, Lourder Torres et al. Rhabdomyosarcoma debut masquerading as acute lymphoblastic leukemia: A case report and review of the literature. Clin. Cae. Rep. 2019; 7:1545.

16. Dong-Hyun Lee, Chan-Jeoung Park, Seongsoo Jang et al. Clinical and Cytogenetic Profiles of Rhabdomyosarcoma with Bone Marrow Involvement in Korean Children: A 15-Year Single-Institution Experience. Ann. Lab. Med. 2018; 38:132-138.

17. Ruymann FB, Newton WA, Jr, Ragab AH et al. Bone marrow metastases at diagnosis in children and adolescents with rhabdomyosarcoma: A report from the Intergroup Rhabdomyosarcoma Study. Cancer 1984; 53:368.

18. Imataki O, Uemura M, Uchida S et al. Complete mimicry: A case of alveolar rhabdomyosarcoma masquerading as acute leukemia. Diagn. Phol. 2017 Nov; 12 (1):77.

19. Yamaguchi K, Koga Y, Suminoe A et al. Alveolar rhabdomyosarcoma of unknown mimicking acute leukemia at the initial presentation. Rinsho Ketsueki 2007 Apr; 48(4):315-20.

20. Ewa Bien, Lucyna Maciejka-Kapuscinska, Maciej Niedzwiecki et al. Childhood rhabdomyosarcoma metastatic to bone marrow presenting with disseminated intravascular coagulation and acute tumor lysis syndrome: Review of the literature apropos of two cases. Clinical and Experimental Metastasis 2010; 27(6):399-407. 